\title{
Legendre coefficients method for linear-quadratic optimal control problems by using genetic algorithms
}

\author{
M. El-Kady, N. El-Sawy* \\ Department of Mathematics, Faculty of Science, Helwan University, Cairo, Egypt \\ Department of Mathematics, Faculty of Science, Alazhar University (girls), Cairo, Egypt \\ *Corresponding author E-mail: nabila_elsawy@yahoo.com
}

\begin{abstract}
In this paper, a numerical method for solving Linear-quadratic optimal control problems (LQPs) is presented. A method is provided for approximating the system dynamics, boundary conditions, and the performance index. The control and state variables are approximated by Legendre orthogonal polynomials. The method is based on using orthogonality of Legendre polynomials to get rid of the integration of the performance index. The problem is then reduced to a constrained optimization problem which is solved by Genetic Algorithms (GAs). Numerical results and comparisons are given at the end of this paper.
\end{abstract}

Keywords: Linear-quadratic optimal control problems; Legendre polynomials; Genetic algorithms.

\section{Introduction}

Optimal control problems (OCPs) pose many problems in engineering which have a large number of applications in many different fields, e.g., mechanical system [2], automotive vehicle design [8] and manufacturing process [6]. Linearquadratic optimal control problems (LQPs) are a class of OCPs and there is an extensive literature about them [11]. The general characterizations of their solutions and useful numerical algorithms to compute them are now available, allowing models with fairly large state spaces, complicated dynamic linkages, and a range of alternative informational assumptions to be handled [5]. In the current study, a review of many papers which give methods for solving LQPs is provided. For example, spectral method [10], time-domain decomposition iterative method [7], and Bézier control points [4]. On the other hand, a substantial literature has discussed the useful notion of orthonormal polynomials such as the use of Legendre polynomials which we have recently dealt with to looking for formulas of Gaussian quadrature.

The aim of this paper is to present a new algorithm for solving LQPs. The proposed algorithm is based on reducing the given OCP to a constrained optimization problem which can be solved by using GAs. This technique aims to use the orthogonal property for Legendre polynomial to eliminate the integration in the performance index without using operational matrix of integration. In order to eliminate the integral operations, the algorithm expands the solution of state $x(t)$ variable and control variable $u(t)$ by a finite series of Legendre polynomials up to degree $N$.

The main advantage for using a Legendre series is the equation error decreases very fast with increasing approximation degree $N$ [14]. Thus, by using few terms of these series, the $L_{2}$-norm of the equation error will presumably become very small.

Our problem is described in $\mathbb{R}$ by the dynamical linear system:

$$
\dot{x}(t)=a(t) x(t)+b(t) u(t), \quad t \in\left[t_{0}, t_{f}\right](1)
$$

The boundary conditions are:

$$
x\left(t_{0}\right)=x_{0}, x\left(t_{f}\right)=x_{f}
$$

The performance measure to be minimized is:

$$
J=\int_{t_{0}}^{t_{f}}\left[q(t) x^{2}(t)+r(t) u^{2}(t)\right] d t
$$


where $(t) \geq 0, r(t)>0$.

Essentially, the LQP formulation (1)-(3) translates the difficulty of the classical control problems, where specifications are typically given in terms of settling times, stability and phase margins, into the choice of the coefficients of the cost matrices. The cost matrix coefficients are approximated in this example, by depending on the observed performance, which is based on Legendre polynomials. Once these matrices are chosen, the approximation of the performance $J$ is free of integration. In practice, GA procedure is typically followed where the properties of the synthesized LQPs are approximated with respect to the given coefficients.

The organization of this paper is as follows: In section 2, we describe the basic formulation of the quadrature and Legendre orthogonal polynomials. In section 3, the Legendre coefficients method for LQPs is presented. We analyze the error upper bounds of the method in section 4. In section 5, GA is presented to solve the resulted optimization problem. Numerical examples are presented in section 6. Finally, conclusions are submitted in section 7.

\section{Quadrature and Legendre orthogonal polynomials}

In this section, some results concerning the Legendre polynomials are introduced. Consider the Legendre Gauss-Lobito (LGL) node points. There is no need for additional nodes with LGL since the endpoint -1 and +1 are collocation points. That is, $t_{0}=-1<t_{1}<\cdots<t_{N}=1$, for $t_{0}=-1, t_{N}=-1$ and $t_{k} \in(-1,1), k=1, \ldots, N-1$ are the zeros of $\dot{P}_{n}(t)$, where $\dot{P}_{n}(t)$, is the derivative of the $n$th order Legendre polynomial $P_{n}(t)$, i.e.

$$
\left\{t_{i}:\left(1-t_{i}^{2}\right) \dot{P}_{n}\left(t_{i}\right)=0, i=0,1, \ldots, N\right\},
$$

where $\left(1-t^{2}\right) \dot{P}_{n+1}(t)=(n+1)\left(P_{n}(t)-t P_{n+1}(t)\right)$.

The three terms of recursive formula are:

$$
P_{n+1}(t)=\frac{2 n+1}{n+1} t P_{n}(t)-\frac{n}{n+1} P_{n-1}(t), \quad n=1,2, \ldots
$$

with initials $P_{0}(t)=1$ and $P_{1}(t)=t$. The orthogonality condition is

$$
\int_{-1}^{1} P_{i}(t) P_{j}(t) d t=\left\{\begin{array}{cc}
\frac{2}{2 i+1}, & i=j, \\
0, & i \neq j .
\end{array}\right.
$$

Suppose that $\boldsymbol{H}=L^{2}[0,1]$ and $\left\{P_{0}, P_{1}, \ldots, P_{m}\right\} \subset \boldsymbol{H}, m \in \mathbb{N} \cup\{0\}$, be the set of Legendre polynomial,

$$
\boldsymbol{Y}=\operatorname{span}\left\{P_{0}, P_{1}, \ldots, P_{m}\right\},
$$

and $f$ be an arbitrary element in $\boldsymbol{H}$. Since $\boldsymbol{Y}$ is a finite-dimensional vector space, $f$ has the unique best approximation out of $Y$ such that for every $y \in Y$ there exist $y_{0} \in \boldsymbol{Y}$;

where $\|f\|_{2}=\sqrt{\langle f, f\rangle}$.

$$
\left\|f-y_{0}\right\|_{2} \leq\|f-y\|_{2}
$$

On the other hand, for $f(t) \in \boldsymbol{Y}$, there exist unique coefficients $c_{j}, j=0, \ldots, m$ such that

$$
f(t)=\sum_{j=0}^{m} c_{j} P_{j}(t)=\boldsymbol{C}^{T} \boldsymbol{\psi},
$$

where $\boldsymbol{\psi}=\left[P_{0}(t), P_{1}(t), \ldots, P_{m}(t)\right]^{T}$ and the coefficients $c_{j}$ are given by

$$
c_{j}=\frac{2 j+1}{2} \int_{-1}^{1} f(t) P_{j}(t) d t, j=1,2, \ldots, m
$$

Now, consider the following assumptions to obtain error estimates:

(i) The function $K$ is Lipschitz with respect to $x$ with Lipschitz constant $L_{M_{x}}$,

i.e.

$$
\left\|K\left(t, x_{1}, u\right)-K\left(t, x_{2}, u\right)\right\| \leq L_{K_{x}}\left\|x_{1}-x_{2}\right\|,
$$


for every $x_{1}, x_{2} \in X, \forall u \in U$ and $\forall t \in[-1,1]$.

(ii) The function $u(t):[-1,1] \rightarrow U$ is satisfied in a Lipschitz condition with Lipschitz $\quad$ constant $L_{u}$

$$
\left\|u\left(t_{1}\right)-u\left(t_{2}\right)\right\| \leq L_{u}\left|t_{1}-t_{2}\right|, \forall t_{1}, t_{2} \in[-1,1]
$$

(iii) The function $K$ is jointly Lipchitz with respect to $x$ and $u$, with Lipschitz constant $L_{M_{x, u}}$, i.e.,

$$
\left\|K\left(t, x_{1}, u_{1}\right)-K\left(t, x_{2}, u_{2}\right)\right\| \leq L_{K_{x, u}}\left\{\left\|x_{1}-x_{2}\right\|+\left\|u_{1}-u_{2}\right\|\right\},
$$

for $\forall x_{1}, x_{2} \in X, \forall u \in U$ and $\forall t \in[-1,1]$.

(iv) Let

$$
\max \left\{\left\|\frac{\partial K(t, x, u)}{\partial t}\right\|, x \in X, u \in U, t \in[-1,1]\right\}=K_{t} .
$$

Suppose that the set $X$ of relevant values of $x(t)$ is defined as follows:

where

$$
X_{\text {rel }}=\left\{x \in \mathbb{R}^{n} ;\|x\| \leq M_{K}\right\}
$$

$$
\max \{\|K(t, x, u)\|, x \in X, u \in U, t \in[-1,1]\}=M_{K} .
$$

The set of $X_{\text {rel }}$ relevant values of $x(t)$ is described generally in [1].

The Legendre coefficients method used in the next section consists of reducing the given LQPs to a set of algebraic equations. We expand the state $x(t)$ and control $u(t)$ variables with the Legendre orthonormal polynomials basis with unknown coefficients. At this moment, the performance index (3) and dynamical system (1) will be in terms of the unknown coefficients.

\section{Legendre coefficients method for LQP}

Consider LQP in equations (1)-(3). The state and control variables have been expand by the Legendre basis $\psi(t)$, respectively, as follows:

where

$$
\begin{aligned}
& D x(t)=\boldsymbol{C}^{T} \dot{\boldsymbol{\psi}}(t), \\
& x(t)=\boldsymbol{C}^{T} \boldsymbol{\psi}(t), \\
& u(t)=\boldsymbol{U}^{T} \boldsymbol{\psi}(t),
\end{aligned}
$$

$$
\begin{aligned}
\boldsymbol{C}^{T} & =\left[c_{0}, c_{1}, \ldots, c_{m}\right], \\
\boldsymbol{U}^{T} & =\left[u_{0}, u_{1}, \ldots, u_{m}\right] .
\end{aligned}
$$

Also by using (5) and (6), we approximated the functions $a(t), b(t), q(t)$ and $r(t)$ by the Legendre basis as follows:

where

$$
\begin{array}{ll}
a(t)=\boldsymbol{A}^{T} \boldsymbol{\psi}(t), & b(t)=\boldsymbol{B}^{T} \boldsymbol{\psi}(t) \\
q(t)=\boldsymbol{Q}^{T} \boldsymbol{\psi}(t), & r(t)=\boldsymbol{R}^{T} \boldsymbol{\psi}(t)
\end{array}
$$

$$
\begin{array}{rlrl}
\boldsymbol{A}^{T} & =\left[a_{0}, a_{1}, \ldots, a_{m}\right], & & \boldsymbol{B}^{T}=\left[b_{0}, b_{1}, \ldots, b_{m}\right], \\
\boldsymbol{Q}^{T}=\left[q_{0}, q_{1}, \ldots, q_{m}\right], & \boldsymbol{R}^{T}=\left[r_{0}, r_{1}, \ldots, r_{m}\right],
\end{array}
$$

with,

$$
\begin{aligned}
& a_{j}=\frac{2 j+1}{2} \int_{-1}^{1} a(t) P_{j}(t) d t, \quad b_{j}=\frac{2 j+1}{2} \int_{-1}^{1} b(t) P_{j}(t) d t, \\
& q_{j}=\frac{2 j+1}{2} \int_{-1}^{1} q(t) P_{j}(t) d t, \quad r_{j}=\frac{2 j+1}{2} \int_{-1}^{1} r(t) P_{j}(t) d t,
\end{aligned}
$$

for $j=0,1, \ldots, m$. 
By using equations (11), (12) and (17), the performance index $J$ can be approximated as:

$$
J_{N}=\int_{-1}^{1}\left[\left(\boldsymbol{Q}^{T} \boldsymbol{\psi}(t)\right) \boldsymbol{C}^{T} \boldsymbol{\psi}(t) \boldsymbol{\psi}(t)^{T} \boldsymbol{C}+\left(\boldsymbol{R}^{T} \boldsymbol{\psi}(t)\right) \boldsymbol{U}^{T} \boldsymbol{\psi}(t) \boldsymbol{\psi}(t)^{T} \boldsymbol{U}\right] d t
$$

The dynamical system (1) is also approximated as follows:

$$
\boldsymbol{C}^{T} \dot{\boldsymbol{\psi}}-\boldsymbol{A}^{T} \boldsymbol{\psi} \boldsymbol{\psi}^{T} \boldsymbol{C}-\boldsymbol{B}^{T} \boldsymbol{\psi} \boldsymbol{\psi}^{T} \boldsymbol{U}=0
$$

The main reason for the use of Legendre orthogonal expansions is that it results in the simplification of the cost function $\mathrm{J}$, this is due to the fact that the integral of the multiplication of non-identical orthogonal terms is zero.

In the following three cases, we studied the integral elimination of performance index:

Case 1: If $q(t)=r(t)=\alpha$ ( $\alpha$ is constant), then the performance index (3) takes the form:

$$
\mathrm{J}_{\mathrm{N}}=\alpha \sum_{j=0}^{N} \frac{2}{2 j+1}\left[c_{j}^{2}+u_{j}^{2}\right]
$$

where $\mathrm{J}_{\mathrm{N}}$ is approximated value of performance index $\mathrm{J}$ due to the approximation of the state and control variables by $N^{\text {th }}$ order Legendre polynomials.

Case 2: If $q(t)=t$ and $r(t)=\alpha$, then the objective function (3) takes the form

where

$$
\left.J_{N}=\sum_{i=0}^{N} \sum_{j=0}^{N} c_{i} c_{j} W_{i j}+\alpha \sum_{j=0}^{N} \frac{2}{2 j+1} u_{j}^{2}\right]
$$

$$
W_{i j}=\int_{-1}^{1} t P_{i} P_{j} d t=\frac{2 \delta_{i j}}{(2 i+1)(2 j+1)}
$$

and

$$
\delta_{i j}=\left\{\begin{array}{cc}
j+1 & , i>j,|i-j|=1 \\
i+1 & , j>i,|i-j|=1 \\
0 & , \text { otherwise }
\end{array}\right.
$$

Case 3: If $q(t)=t^{2}$ and $r(t)=\alpha$ then the objective function (3) takes the form:

where

$$
\left.J_{N}=\sum_{i=0}^{N} \sum_{j=0}^{N} c_{i} c_{j} E_{i j}+\alpha \sum_{j=0}^{N} \frac{2}{2 j+1} u_{j}^{2}\right]
$$

and

$$
E_{i j}=\int_{-1}^{1} t^{2} P_{i} P_{j} d t=2 \delta_{i j}
$$

$$
\delta_{i j}=\left\{\begin{array}{cc}
\frac{j(j+1)}{\left(4 j^{2}-1\right)(2 j+3)} & , i>j,|i-j|=2 \\
\frac{1}{(2 i+1)^{2}\left[\frac{(i+1)^{2}}{2 i+3}+\frac{i^{2}}{2 i-1}\right]}, i=j \\
\frac{i(i+1)}{\left(4 i^{2}-1\right)(2 i+3)} \quad, j>i,|i-j|=2 \\
0 \quad, \text { otherwise }
\end{array}\right.
$$


The algorithm that solves the LQP in equations (1)-(3) is shown in Table 1.

\section{Error estimation}

We denote the error in $x(t)$ by $e=\left\|x-x_{m}\right\|$.

\subsection{Error estimation for the derivative of Legendre polynomial}

The calculated error of the derivatives of Legendre polynomials can be obtained as follows: [12]

$$
\left\|e_{D_{i}}\right\|_{2} \leq(2 i+1) \sum_{k=1}^{i}\left|\frac{(i+k) !}{(i-k) ! k !(k-1) !}\right|\left(\frac{2^{m+1} \Gamma\left(\frac{3}{2}+m\right)}{\sqrt{\pi}}\right)^{\frac{1}{2}} G\left(t^{k-1}, P_{0}, \ldots, P_{m}\right),
$$

$0 \leq i \leq m$.

where $e_{D_{i}}$ is the upper bound error for of the differentiation matrix $D$ in terms of Gram determinant $G\left(t^{k-1}, P_{0}, \ldots, P_{m}\right)$. [3].

\subsection{Error estimation in the dynamic system}

Assume $([-1,1],\|\|$.$) to be the space of all continuous functions with the norm:$

Let $\|f(t)\|=\max |f(t)|) \forall t \in[-1,1]$.

$$
\max \left\{\left\|K\left(t_{1}, x_{m}, u_{m}\right)-K\left(t_{2}, x, u\right)\right\|, x_{m} \in X, u_{m} \in U, t_{1}, t_{2} \in[-1,1]\right\}=M_{K_{m}} .
$$

Under the above hypothesis in (7)-(10), we have the following theorem.

Theorem 4.1: If the conditions (7)-(10) are satisfied, then for every $u(t) \in U$, the error estimate in Eq. (1) satisfies: where

$$
\left\|x-x_{m}\right\| \leq \sigma+\bar{H}
$$

$$
\sigma=\alpha+M_{K_{m}}
$$

Proof: Let $\alpha=\left\|\dot{x}-\dot{x}_{m}\right\|$, we have

$$
\bar{H}=K_{t}+L_{K_{x, u}}\left(K_{x}+K_{u}\right)
$$

$$
\begin{aligned}
&\left\|x-x_{m}\right\|=\max \mid x(t)-x_{m}(t) \mid \\
& \leq \alpha+\max \left(\left\|K(t, x(t), u(t))-K\left(t, x_{m}(t), u_{m}(t)\right)\right\|\right) \\
& \leq \alpha+\max \left(\left\|K(t, x(t), u(t))-K\left(t_{i}, x_{m}(t), u_{m}(t)\right)\right\|\right. \\
&+\left\|K\left(t_{i}, x(t), u(t)\right)-K\left(t_{i}, x_{m}\left(t_{i}\right), u_{m}\left(t_{i}\right)\right)\right\| \\
&\left.+\left\|K\left(t_{i}, x_{m}\left(t_{i}\right), u_{m}\left(t_{i}\right)\right)-K\left(t, x_{m}(t), u_{m}(t)\right)\right\|\right) \\
& \leq \alpha+M_{K_{m}}+\max \left(\left(K_{t}+L_{K_{x, u}}\left(M_{K}+L_{u}\right)\right)\right. \\
& \leq \sigma+\bar{H}
\end{aligned}
$$

for sufficiently small $\sigma$ the proof is complete.

\subsection{Error estimation of objective functional}

Now for the purpose of error estimation in Eq. (3), we suppose the following conditions:

(i) Let

$$
\max \left\{\left\|\frac{\partial F(t, x, u)}{\partial t}\right\|, x \in X, u \in U, t \in[-1,1]\right\}=F_{t} .
$$

(ii)

$$
\text { Let }
$$


(iii)

$$
\max \left\{\left\|F\left(t_{1}, x_{m}, u_{m}\right)-F\left(t_{2}, x, u\right)\right\|, x_{m} \in X, u_{m} \in U, t_{1}, t_{2} \in[-1,1]\right\}=M_{F_{m}} .
$$

(iv) The function $F$ is jointly Lipschitz with respect to $x$ and $u$, with Lipschitz constant $L_{F_{x, u}}$,

(v)

$$
\left\|F\left(t_{1}, x_{m}, u_{m}\right)-F\left(t_{2}, x, u\right)\right\| \leq L_{F_{x, u}}\left\{\left\|x_{1}-x_{2}\right\|+\left\|u_{1}-u_{2}\right\|\right\}
$$

For every $x_{1}, x_{2} \in X, \forall u_{1}, u_{2} \in U$ and $\forall t \in[-1,1]$.

Theorem 4.2: If the conditions (23)-(25) are satisfied, then for every $u(t) \in U$, the error estimation in Eq. (20) satisfies: where

$$
\left\|J-J_{m}\right\| \leq \gamma+\bar{F}
$$

$$
\begin{aligned}
& \gamma=2 M_{F_{m}}, \\
& \bar{F}=F_{t}+L_{F_{x, u}}\left(M_{x}+M_{u}\right) .
\end{aligned}
$$

Proof: Consider:

$$
\begin{gathered}
\left\|J-J_{m}\right\|=\max \left|J(x(t), u(t))-J_{m}(x(t), u(t))\right| \\
\leq \max \left(\int_{-1}^{1}\left\|F(t, x(t), u(t))-F\left(t, x_{m}(t), u_{m}(t)\right)\right\| d t\right) \\
\leq \max \left(\int_{-1}^{1}\left\|F(t, x(t), u(t))-F\left(t_{i}, x_{m}(t), u_{m}(t)\right)\right\| d t+\int_{-1}^{1}\left\|F\left(t_{i}, x(t), u(t)\right)-F\left(t_{i}, x_{m}\left(t_{i}\right), u_{m}\left(t_{i}\right)\right)\right\| d t\right. \\
\left.+\int_{-1}^{1}\left\|F\left(t_{i}, x_{m}\left(t_{i}\right), u_{m}\left(t_{i}\right)\right)-F\left(t, x_{m}(t), u_{m}(t)\right)\right\| d t\right) \\
\leq 2 M_{F_{m}}+\max \left(\left(F_{t}+L_{F_{x, u}}\left(M_{x}+M_{u}\right)\right) \int_{-1}^{1}\left|t-t_{i}\right| d t\right. \\
\leq \gamma+\bar{F}
\end{gathered}
$$

for sufficiently small $\gamma$ the proof is complete.

\section{Implementation of genetic solver for LQP}

Genetic Algorithm (GA) is a heuristic search technique based on the evolutionary ideas of natural selection. It is usually used to solve optimization problem of the form:

$\max (\min ) f(x)$

Subject to $x \in M$.

The function $f: R^{n} \rightarrow \mathrm{R}$ is the objective function and the set $M \subset R^{n}$ is the feasible set of (26). Based on the description of the function $f$ and the feasible set $M$, the problem (26) can be classified as linear, quadratic, non-linear, semi-infinite, semi-definite, multiple-objective, discrete optimization problem etc.

The algorithm to solve a problem must have five components [9, 13]:

1. A genetic representation of solutions to the problem,

2. A way to create an initial population of solutions,

3. An evaluation function that plays the role of the environment, rating solutions in terms of their "fitness",

4. Genetic operators that alter the genetic composition of children during reproduction,

5. Values for the parameters that the GA use (population size, probabilities of applying genetic operators, etc.).

Table 2 shows the code of the general GA algorithm.

In GA, there are many parameters and operators that have to be adjusted. The following parameters should be carefully chosen: namely, Population type, Population size, Crossover rate, Mutation rate, Selection operator and Crossover operator. The parameters adopted in the implementation of the proposed algorithm are listed in Table 3. 
Table 1. LQP algorithm

$\underline{L Q P \text { algorithm }}$

Step (0) Initialization: Set $k=0$, choose $N, \varepsilon$.

Step (1) Use the approximation of the state variable $x(t)$ and the control variable $u(t)$ as in equations (11)-(13).

Step (2) Eliminate the integration the performance index as in cases 1-3.

Step (3) Solve the constrained optimization problem by GA.

Step (4) Stop if :

Otherwise, $k=k+1$ and go to Step (3).

$$
\left|J_{N+1}-J_{N}\right| \leq \varepsilon
$$

\section{Table 2. GA algorithm}

$\underline{\text { GA algorithm }}$

Generate an initial population;

Evaluate fitness of individuals in the population;

Do:

Select parents from population;

Recombine (mate(crossover and mutation operators)) parents to

produce children;

Evaluate fitness of the children;

Replace some or all of the population by the children;

while a satisfactory solution has been found.

Table 3.The algorithm parameters.

Population type

Population size

Crossover rate

Mutation rate

Selection operator

Crossover operator double Vector

100

0.9

0.1

Stochastic uniform Scattered

\section{Numerical examples}

In this section, we apply the proposed method to solve LQPs. Numerical results of these examples are given to clarify the accuracy of the proposed method. All computations were carried out by MATLAB 2010a.

Example (1): Consider the following LQP:

Minimize

Subject to

$$
J=\frac{1}{2} \int_{-1}^{1}\left(x^{2}(t)+u^{2}(t)\right) d t
$$

$$
\begin{aligned}
2 \dot{x}(t) & =u(t), \text { and } \\
x_{1}(-1) & =\frac{1+3 e}{2(1-e)} .
\end{aligned}
$$

By applying the Legendre coefficients method, the problem can be converted to the following constrained optimization problem: Minimize

Subject to

$$
J_{N}=\frac{1}{2} \sum_{j=0}^{N} \frac{2}{2 j-1}\left(c_{j}^{2}+u_{j}^{2}\right)
$$




$$
2 \sum_{j=0}^{N} c_{j} \dot{P}_{j}\left(t_{i}\right)-\sum_{j=0}^{N} u_{j} P_{j}\left(t_{i}\right)=0, \quad i=0, \ldots, N
$$

and

$$
x_{1}(-1)=\frac{1+3 e}{2(1-e)}
$$

Legendre coefficients method gives the following numerical solution:

$$
\begin{gathered}
x=-1.946+0.449 t-0.244 t^{2}+0.02 t^{3}-0.004 t^{4}, \\
u=0.899-0.973 t+0.114 t^{2}-0.04 t^{3} .
\end{gathered}
$$

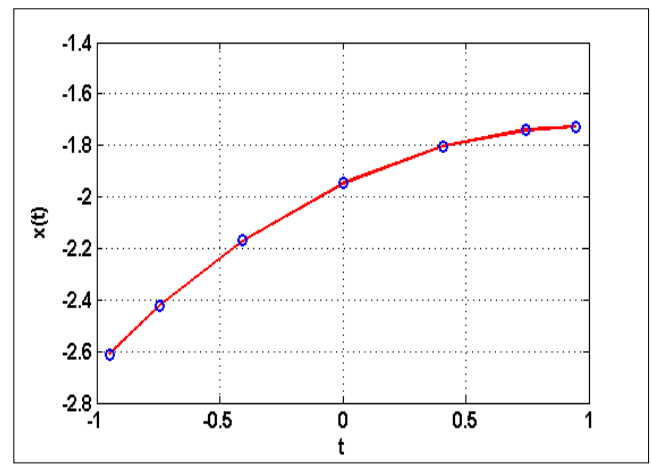

\begin{tabular}{|c|c|c|c|c|}
\hline \multirow{2}{*}{$\mathrm{N}$} & \multirow{2}{*}{$\begin{array}{c}\text { No. of } \\
\text { unknowns }\end{array}$} & \multicolumn{2}{|c|}{$J^{*}$ calculated by GA method } & \multirow{2}{*}{$\begin{array}{l}J^{*} \text { calculated by } \\
\text { Penalty method }\end{array}$} \\
\hline & & No. of iteration & $J^{*}$ No. & \\
\hline \multirow{4}{*}{4} & \multirow{4}{*}{10} & 50 & 5.4002 & \multirow{4}{*}{5.4048} \\
\hline & & 100 & 5.3990 & \\
\hline & & 500 & 5.3990 & \\
\hline & & 1000 & 5.3988 & \\
\hline \multirow{4}{*}{6} & \multirow{4}{*}{14} & 50 & 5.4002 & \multirow{4}{*}{5.4048} \\
\hline & & 100 & 5.3991 & \\
\hline & & 500 & 5.3990 & \\
\hline & & 1000 & 5.3989 & \\
\hline \multirow{4}{*}{8} & \multirow{4}{*}{18} & 50 & 5.4004 & \multirow{4}{*}{5.4048} \\
\hline & & 100 & 5.3996 & \\
\hline & & 500 & 5.3989 & \\
\hline & & 1000 & 5.3990 & \\
\hline
\end{tabular}

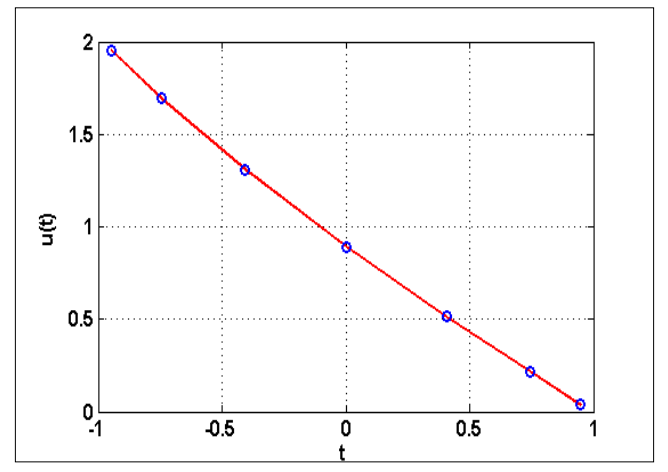

Fig.1: The state $\mathrm{x}(\mathrm{t})$ and control $\mathrm{u}(\mathrm{t})$ variables for Example (1) with $\mathrm{N}=6$

Table 4: Results provided by Penalty method and our approach of Example (1)

In Table 4, the Legendre coefficients method with GA is applied to Example (1) with different values of N. It is obvious that with increase in the number of the iteration our approach has a successful rate of minimization more than the Penalty method. Finally, Fig.1 present the optimal states and control variables.

Example (2) Consider the following LQP:

Minimize

$$
J=\frac{1}{4} \int_{-1}^{1}\left(x_{1}^{2}(t)+u^{2}(t)\right) d t
$$

Subject to

$$
\begin{gathered}
2 \dot{x}_{1}(t)=x_{2}(t) \\
2 \dot{x}_{2}(t)=-x_{2}(t)+u(t)
\end{gathered}
$$

and

$$
x_{1}(-1)=0, x_{2}(-1)=10 \text {. }
$$

By applying the procedure described here, the problem can be converted to the following constrained optimization problem:

Minimize 
Subject to

$$
J_{N}=\frac{1}{4} \sum_{j=0}^{N} \frac{2}{2 j+1}\left(c_{j}^{2}+u_{j}^{2}\right)
$$

$$
\begin{gathered}
2 \sum_{j=0}^{N} c_{j} \dot{P}_{j}\left(t_{i}\right)-\sum_{j=0}^{N} b_{j} P_{j}\left(t_{i}\right)=0, i=0, \ldots, N \\
2 \sum_{j=0}^{N} b_{j} \dot{P}_{j}\left(t_{i}\right)+\sum_{j=0}^{N} b_{j} P_{j}\left(t_{i}\right)-\sum_{j=0}^{N} u_{j} P_{j}\left(t_{i}\right)=0, i=0, \ldots, N
\end{gathered}
$$

and

$$
x_{1}(-1)=0, x_{2}(-1)=10 \text {. }
$$

By using Legendre coefficients method, we get:

$$
\begin{gathered}
x_{1}=3.804+2.826 \mathrm{t}-0.822 t^{2}+0.170 t^{3}+0.026 t^{4}, \\
x_{2}=5.652-3.111 t+1.026 t^{2}-0.21 t^{3}, \\
u=-0.568+0.995 t-0.234 t^{2}-0.21 t^{3} .
\end{gathered}
$$

\begin{tabular}{|c|c|c|c|c|}
\hline \multirow{2}{*}{$\mathrm{N}$} & \multirow{2}{*}{$\begin{array}{c}\text { No. of } \\
\text { unknowns }\end{array}$} & \multicolumn{2}{|c|}{$J^{*}$ calculated by GA method } & \multirow{2}{*}{$\begin{array}{l}J^{*} \text { calculated by } \\
\text { Penalty method }\end{array}$} \\
\hline & & No. of iteration & $J^{*}$ & \\
\hline \multirow{4}{*}{4} & \multirow{4}{*}{10} & 50 & 8.0460 & \multirow{4}{*}{8.0514} \\
\hline & & 100 & 8.0454 & \\
\hline & & 500 & 8.0434 & \\
\hline & & 1000 & 8.0434 & \\
\hline \multirow{4}{*}{6} & \multirow{4}{*}{14} & 50 & 8.0486 & \multirow{4}{*}{8.0514} \\
\hline & & 100 & 8.0436 & \\
\hline & & 500 & 8.04340 & \\
\hline & & 1000 & 8.04334 & \\
\hline \multirow{4}{*}{8} & \multirow{4}{*}{18} & 50 & 8.0459 & \multirow{4}{*}{8.0514} \\
\hline & & 100 & 8.0449 & \\
\hline & & 500 & 8.0439 & \\
\hline & & 1000 & 8.0439 & \\
\hline
\end{tabular}
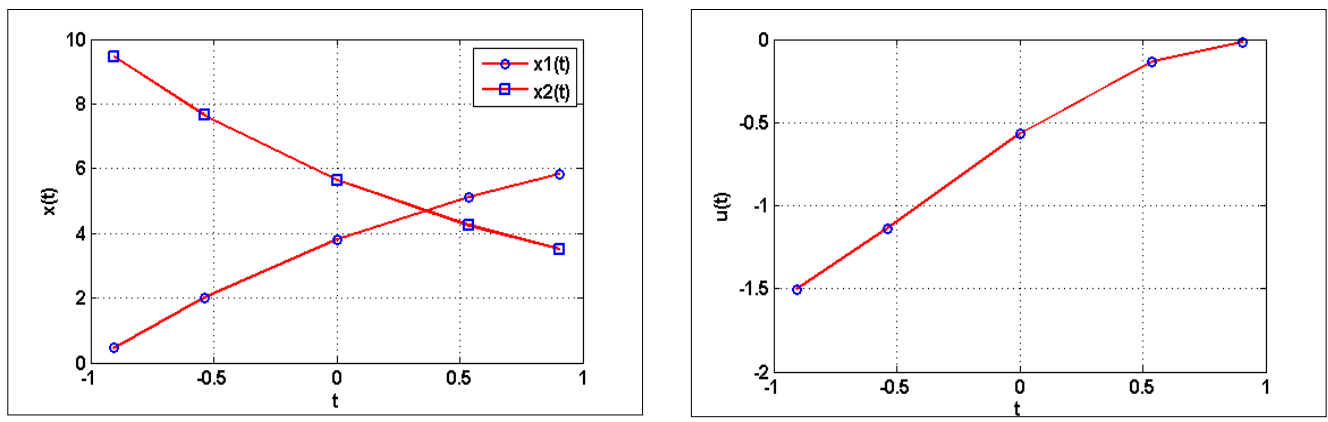

Fig.2: Values of states $\mathrm{x}(\mathrm{t})$ and control $\mathrm{u}(\mathrm{t})$ variables for Example(2) with $\mathrm{N}=4$

In Table 5, we note that using the GA reaches a near-optimal solution when a reasonable number of iterations increase. Fig. 2 shows the values of states and control variables with $\mathrm{N}=4$.

Example (3): Consider the following LQP:

Minimize

$$
J=\frac{1}{4} \int_{-1}^{1}\left(x^{2}(t)+u^{2}(t)\right) d t
$$


Subject to

$$
\begin{gathered}
2 \dot{x}(t)=t x(t)+u(t), \text { and } \\
x_{1}(-1)=1 .
\end{gathered}
$$

By applying the Legendre coefficients method, the problem can be converted to the following constrained optimization problem: Minimize

Subject to

$$
J_{N}=\frac{1}{4} \sum_{j=0}^{N} \frac{2}{2 j-1}\left(c_{j}^{2}+u_{j}^{2}\right)
$$

$$
2 \sum_{j=0}^{N} c_{j} \dot{P}_{j}\left(t_{i}\right)-t_{i} \sum_{j=0}^{N} c_{j} x_{j} P_{j}\left(t_{i}\right)-\sum_{j=0}^{N} u_{j} P_{j}\left(t_{i}\right)=0, \quad i=0, \ldots, N
$$

and

$$
x_{1}(-1)=1 .
$$

Legendre coefficients method gives the following numerical solution:

$$
\begin{gathered}
x=0.577-0.156 t+0.214 t^{2}-0.225 t^{3}+0.031 t^{4}, \\
u=-0.312+0.287 t+0.041 t^{2}-0.008 t^{3}-0.009 t^{4} .
\end{gathered}
$$

\begin{tabular}{|c|c|c|c|c|}
\hline \multirow{2}{*}{$\mathrm{N}$} & \multirow{2}{*}{$\begin{array}{c}\text { No. of } \\
\text { unknowns }\end{array}$} & \multicolumn{2}{|c|}{$J^{*}$ calculated by GA method } & \multirow{2}{*}{$\begin{array}{l}J^{*} \text { calculated by } \\
\text { Penalty method }\end{array}$} \\
\hline & & No. of iteration & $J^{*}$ & \\
\hline \multirow{4}{*}{4} & \multirow{4}{*}{10} & 50 & 0.2795 & \multirow{4}{*}{0.2802} \\
\hline & & 100 & 0.2794 & \\
\hline & & 500 & 0.2794 & \\
\hline & & 1000 & 0.2794 & \\
\hline \multirow{4}{*}{6} & \multirow{4}{*}{14} & 50 & 0.2793 & \multirow{4}{*}{0.2802} \\
\hline & & 100 & 0.2793 & \\
\hline & & 500 & 0.2793 & \\
\hline & & 1000 & 0.2793 & \\
\hline \multirow{4}{*}{8} & \multirow{4}{*}{18} & 50 & 0.2796 & \multirow{4}{*}{0.2802} \\
\hline & & 100 & 0.2794 & \\
\hline & & 500 & 0.2793 & \\
\hline & & 1000 & 0.2793 & \\
\hline
\end{tabular}
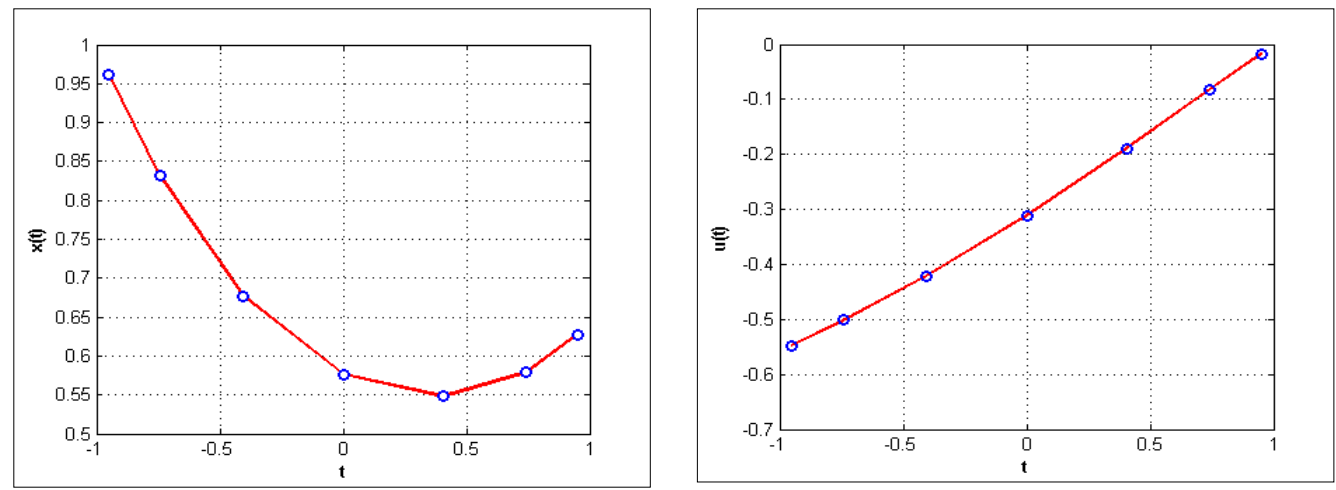

Fig. 3: The state $\mathrm{x}(\mathrm{t})$ and control $\mathrm{u}(\mathrm{t})$ variables for Example (3) with $\mathrm{N}=6$

Table 6: Results provided by Penalty method and our approach of Example (3)

In Table 6, we obvious that with increasing the number of the iteration our approach has a successful rate of minimization more than the Penalty method. Fig.3 present the optimal states and control variables. 


\section{Conclusion}

A numerical method for solving LQPs with terminal state constraints on the states and controls has been presented. The method is based on the orthogonality property of Legendre approximations. Therefore, our method prevents the need for solving the backward integration of the matrix Riccati differential equation or inverting ill-conditioned transition matrices. The method replaces the constrained OCP by a quadratic programming which is solved by using GAs. GAs have a distinguishing feature which makes them very effective when considering linear optimization. GAs can be especially effective when solving highly linear models. On the other hand GAs can also be used to provide quick, near global-optimal solution. To sum up, it is the final aim of the study to achieve the optimal strategy and optimal trajectory.

\section{References}

[1] S.A. Belbas, A new method for optimal control of Volterra integral equations, Appl. Math. Comput. 189 (2007) 1902-1915.

[2] Y. J. Cho, M. Matic, J. Pečaric, Josip, On Gram's determinant in 2-inner product spaces, J. Korean Math. Soc. 38 (6) (2001) $1125-1156$.

[3] Fábio Roberto Chavarette, José Manoel Balthazar, Jorge L.P. Felix, Marat Rafikov, A reducing of a chaotic movement to a periodic orbit, of a micro-electro-mechanical system, by using an optimal linearcontrol design, Commun. Nonlinear Sci. Numer. Simul. 14 (2009) $1844-1853$.

[4] Mortaza Gachpazan, Solving of time varying quadratic optimal control problems by using Bézier control points, Comput. \& Appl. Math. 30 (2011) 367-379.

[5] Gerali, Andrea, F. Lippi, Solving Dynamic Linear-Quadratic Problems with Forward-Looking Variables and Imperfect Information using Matlab, unpublished, Bank of Italy, November,2005.

[6] Rodolfo E. Haber, Rǻl M. del Toro, Agustín Gajate, Optimal fuzzy control system using the cross-entropy method. A case study of a drilling process , Information Sciences. 180 (2010) 2777-2792.

[7] M. Heinkenschloss, A time-domain decomposition iterative method for the solution of distributed linear quadratic optimal control problems, J. Comput. \& Appl. Math. 173 (2011) 169-198.

[8] N. Jalili, E. Esmalzadeh, Optimum active vehicle suspensions with actuator time delay, transactions of the ASME, J. Dyn. Syst., Measur. Control 123 (2001) 54-61

[9] Jianmin H, Xiaoping Hu, Hongsheng Lue, Nonstandard optimal control by utilizing genetic algorithms ,World Journal of Modelling and Simulation. 1(2) (2005)75-80

[10] H. Juddu, Spectral method for constrained linear-quadratic optimal control. Math. Comput. Simul. 58 (2002) $159-169$.

[11] Kendrick, David A, Stochastic Control for Economic Models: Past, Present and the Paths Ahead, J. Econ. Dynam. Control. 29(2005) 3-30.

[12] A. Lotfi, Mehdi Dehghan, S.A. Yousefi , A numerical technique for solving fractional optimal control problems, Comput. Math. Appl. 62 (2011) 1055-1067.

[13] A.K. Maity, K. Maity, S. Mondal, M. Maiti, A Chebyshev approximation for solving the optimal production inventory problem of deteriorating multi-item, Math. Comput. Modell. 45( 2007) 149-161.

[14] J. Vlassenbroeck, A Chebyshev polynomial method for optimal control with state constraints, Automatica 24 (1988) $499-506$. 